\title{
REVIEW \\ Genetic and Breeding Analysis of Blast Resistance in Elite Indica-type Rice (Oryza sativa L.) Bred in International Rice Research Institute
}

\author{
Yoshimichi FUKUTA ${ }^{1 *}$, Leodegario A. EBRON ${ }^{2}$ and Nobuya KOBAYASHI ${ }^{2}$ \\ ${ }^{1}$ Biological Resources Division, Japan International Research Center for Agricultural Sciences \\ (JIRCAS) (Tsukuba, Ibaraki 305-8686, Japan) \\ ${ }^{2}$ Plant Breeding, Genetics, and Biotechnology Division, International Rice Research Institute (IRRI) \\ (DAPO Box 7777, Metro Manila, Philippines)
}

\begin{abstract}
Using a new differential system which is based on the gene-for-gene relationship between rice resistance genes and blast races, at least seven kinds of genes, Pi20, Pita, Pik (one of the Pik allele genes except for Pik-s), Pik-s, Pib, Piz-t, and Pii or Pi3, were estimated in 42 International Rice Research Institute (IRRI)-bred rice (Oryza sativa $\mathrm{L}$.) varieties using 14 blast isolates from the Philippines. Moreover, genetic analyses based on the segregation of $\mathrm{BC}_{1} \mathrm{~F}_{2}$ populations derived from the crosses between IRRI varieties and susceptible rice, $\mathrm{CO} 39$, as recurrent parents, and allelism tests with differential varieties, were performed to identify and confirm these estimated genes using 10 varieties among them. A total of 7 genes, Pi20, Pita, Pik ${ }^{\dagger}$, Pia, Pib, Pik-s, and Piz-t, were identified. In some varieties, the four genes $P i a, P i b, P i k-s$, and Piz-t that were not estimated by reaction patterns in the previous analysis, were identified by these genetic analyses. Among the genes identified, Pib and Pik alleles (Pik-s or $P i k^{\dagger}$ ) were considered widely distributed.
\end{abstract}

Discipline: Plant breeding

Additional key words: IRRI-Japan collaborative research project, monogenic line, plant protection, reaction pattern

\section{Introduction}

Blast disease, caused by Pyricularia grisea Sacc., is a serious disease of rice worldwide. The use of host resistance offers the most economical and effective means of controlling blast disease.

A total of 46 Indica-type inbreds and two hybrid varieties were developed by the International Rice Research Institute (IRRI) and released for irrigated lowland rice systems by $1999^{22}$. These varieties and their derivatives have been widely grown in South and Southeast Asia and account for more than $80 \%$ of total rice production in this region ${ }^{13}$. Among these varieties, IR8, released in 1966, triggered the Green Revolution in tropical countries of Asia ${ }^{7}$. IR36 once dominated rice pro- duction in several Asian countries in the $1970 \mathrm{~s}^{11}$. Released in 1985, IR64 has been widely accepted as a high-quality rice variety in many countries because of its desirable combination of intermediate amylose content and intermediate gelatinization temperature ${ }^{12}$. A more recent variety, IR72, displays a high yield potential, shorter growth duration, and improved resistance to several diseases and insect pests ${ }^{17}$.

In spite of the wide distribution of many known genes in rice varieties grown in different countries, genetic studies of blast resistance are limited in the tropics. There were only a few of these studies that were conducted in Indica-type varieties particularly those bred in IRRI. Flores-Gaxiola et $\mathrm{al}^{4}$ reported two complementary genes and one dominant gene in the three progenies derived from a cross of IR8 with Tetep. Yu et al. ${ }^{33}$ showed that the

This study was carried out under the IRRI-Japan collaborative research project, phases III, IV and V, which were supported by the Ministry of Agriculture, Forestry and Fisheries of Japan. This is a review of genetic analyses for blast resistance genes in IRRI-bred rice varieties, which were already published in some scientific reports ${ }^{1,2,9}$.

*Corresponding author: e-mail zen@jircas.affrc.go.jp

Received 15 February 2006; accepted 19 September 2006. 
resistance of five varieties, IR36, IR46, IR54, IR56, and IR60, was generally controlled by one or two dominant genes. They also recognized a recessive gene that controlled resistance in IR54 against one blast isolate. Yamaguchi et al. ${ }^{29}$ reported two genes, Pia and Pib, in IR50. Imbe et al. ${ }^{10}$ reported that four genes, Pia, Pib, Pik$s$, and Pi20, are harbored in IR24. Popularly known IR36 has shown a high level of partial resistance ${ }^{23,31}$ which was attributed to a number of minor genes ${ }^{27}$. It was also shown to carry at least three major genes ${ }^{33}$ for complete resistance but the genotypes for blast resistance were not known. Five new genes were identified in IR64 using a QTL strategy on a set of doubled-haploid lines (DHL) ${ }^{24}$. Thus, these studies have provided only fragmentary knowledge, and have not been the result of a survey to research the resistance genes in IRRI-bred rice varieties.

There are several factors for these limited studies, one of which was attributed to the lack of a suitable differential system for efficient identification of blast resistance genes. Resistance to blast follows a gene-for-gene system $^{3}$. On this basis, the resistance genotypes of a variety can be inferred by their reaction patterns to blast isolates with known avirulence or virulence to resistance genes. An important prerequisite is the selection of suitable isolates that can discriminate resistance genes. An avirulent isolate is necessary to identify a resistance gene that would otherwise escape detection ${ }^{20}$. The Japanese differential varieties ${ }^{16,28}$ may have single resistance genes to Japanese isolates, but these varieties are not well adapted for tropical blast isolates. They have additional gene(s) which mask the target gene's reaction ${ }^{19}$. For instance, the Pish gene $^{8}$ carried by several Japanese differentials was not discovered for many years after its intensive use because no isolate avirulent to Pish was observed during that time.

This problem has been recently addressed with the development of a differential system for blast resistance genes involving Philippine blast isolates. Tsunematsu et al. ${ }^{26}$ and Fukuta et al. ${ }^{5}$ developed the monogenic lines as the differential varieties containing 24 known blast resistance genes in IRRI. Each of these lines carries a single resistance gene developed in the genetic background of Japonica-type variety Lijiang-xin-tuan-heigu (LTH).

Using the differential system consisting of monogenic lines and differential blast isolates from the Philippines, the presence and kinds of blast resistance genes in IRRI-bred rice varieties were estimated. Moreover, segregation analyses and allelism tests were also carried out to identify and confirm these genes.

\section{Material and methods}

\section{IRRI-bred rice varieties}

A total of 42 rice (Oryza sativa L.) varieties bred at IRRI were used for estimating the blast resistance genes (Table 1). The widely distributed varieties IR8, IR24, IR36, IR64, and IR72, which have outstanding traits such as high yield or grain quality, and used in many countries, were included. Eight varieties, PSBRc1, PSBRc2, PSBRc4, PSBRc10, PSBRc18, PSBRc20, PSBRc28, and PSBRc30, developed by IRRI and designated by the Philippine Seed Board were also included.

\section{Inoculation and evaluation of resistance}

Seven seeds for each variety were sown in a $26 \times 35$ $\mathrm{cm}$ plastic tray with two replications. In all cases, two varieties, CO39 and Lijiang-xin-tuan-heigu (LTH), were placed in each tray to determine the success of inoculation and virulence of blast isolates. At the fourth-leaf stage, these seedlings in each tray were sprayed with $40-50 \mathrm{~mL}$ of spore suspension adjusted to $10^{5}$ spores $\mathrm{mL}^{-1}$. These seedlings were placed inside wet jute sacks for 18-24 h and transferred to an air-conditioned glasshouse room with $23 \pm 3^{\circ} \mathrm{C} / 30 \pm 5^{\circ} \mathrm{C}$ night and day temperatures. Disease reaction of each seedling was examined 6-7 days after inoculation. The reaction was evaluated using a $0-5$ scale with slight modifications as described by Mackill and Bonman ${ }^{18}$ in which $0=$ no evidence of infection; $1=$ brown specks smaller than $0.5 \mathrm{~mm}$ in diameter; 2 = brown specks about $0.5-1.0 \mathrm{~mm}$ in diameter; $3^{-}=$roundish to slightly elliptical lesions about $1-2 \mathrm{~mm}$ in diameter with gray centers surrounded by brown margins; $3^{\mathrm{m}}=$ lesions found only at the leaf margins; $3^{+}=$lesions $2-3 \mathrm{~mm}$ in length, and 1-2 $\mathrm{mm}$ in breadth with gray centers surrounded by brown margins; 4 = typical spindle-shaped lesions more than $3 \mathrm{~mm}$ in diameter and more than $2 \mathrm{~mm}$ in breadth with necrotic gray centers and reddish brown margins; 5 = typical spindle-shaped lesions more than 3 $\mathrm{mm}$ in diameter or more than $2 \mathrm{~mm}$ in breadth with necrotic gray centers and water-soaked or yellowish margins; and $5^{+}=$upper portion of one or two leaves killed by coalescence of lesions with type 5 lesions. Scores of 0-2 were evaluated as resistant (R); $3^{-}$and $3^{\mathrm{m}}$ as moderately resistant (MR); $3^{+}$as moderately susceptible (MS); and finally 4,5 and $5^{+}$as susceptible (S). Fourteen blast isolates PO6-6, IK81-25, M36-1-3-10-1, IK81-3, BN111, Ca89, V850256, M39-1-3-8-1, M64-1-3-9-1, M39-1-221-2, BN209, V850196, B90002, and C923-49 that were previously selected by Yanoria et al. ${ }^{30}$, were used for the inoculation. 
Table 1. List of IRRI-bred rice varieties used for identifying the blast resistance genes

\begin{tabular}{|c|c|c|c|c|}
\hline Variety & $\begin{array}{l}\text { Year of } \\
\text { release }\end{array}$ & $\begin{array}{l}\text { Environment } \\
\text { suited }\end{array}$ & Line designation & Cross combination \\
\hline IR5 & 1967 & Irrigated & IR5-47-2 & Peta/tangkai Rotan \\
\hline IR8 & 1966 & Irrigated & IR8-288-2 & Pata/Dee-geo-woo-gen \\
\hline IR20 & 1969 & Irrigated & IR523-E576 & IR262-24-3/TKM6 \\
\hline IR22 & 1969 & Irrigated & IR579-160-2 & IR8/Tadukan \\
\hline IR24 & 1971 & Irrigated & IR661-1-140-3 & IR8/IR127-2-2 \\
\hline IR26 & 1973 & Irrigated & IR1541-102-7 & IR24/TKM6 \\
\hline IR28 & 1974 & Irrigated & IR2061-214-3-8-2 & IR833///IR1561-149-1//IR24*4/O. nivara \\
\hline IR29 & 1974 & Irrigated & IR2061-464-4-14-1 & IR833///IR1561-149-1//IR24*4/O. nivara \\
\hline IR30 & 1974 & Irrigated & IR2153-159-1-4 & IR1541-102-6-3//IR24*4/O. nivara \\
\hline IR32 & 1975 & Irrigated & IR2070-747-6-3-2 & IR20*2/O. nivara//CR94-13 \\
\hline IR34 & 1975 & Irrigated & IR2061-213-2-17 & IR833-6-2-1-1//IR1561-149-1//IR24*4/O. nivara \\
\hline IR36 & 1976 & Irrigated & IR2071-625-1-252 & IR1561-228-1-2/IR737//CR94-13 \\
\hline IR38 & 1976 & Irrigated & IR2070-423-2-5-6 & IR20*2/O. nivara//CR94-13 \\
\hline IR40 & 1977 & Irrigated & IR2070-414-3-9 & IR20*2/O. nivara//CR94-13 \\
\hline IR42 & 1977 & Irrigated & IR2071-586-5-6-3 & IR1561-228-1-2/IR737//CR94-13 \\
\hline IR43 & 1978 & Irrigated & IR1529-430-3 & IR305-3-17-1-3/IR661-1-140-3 \\
\hline IR44 & 1978 & Irrigated & IR2863-38-1-2 & IR1529-680-3/CR94-13//IR480-5-9-3 \\
\hline IR45 & 1978 & Irrigated & IR2035-242-1 & IR1416-128-5/IR1364-37-3-1//IR1824-1 \\
\hline IR46 & 1978 & Irrigated & IR2058-78-1-3-2 & IR1416-128-5/IR1364-37-3-1//IR1366-120-3-1/IR1539-111 \\
\hline IR48 & 1979 & Irrigated & IR4570-83-3-3 & IR1702-74-3-2/IR1721-11-6-8-3//IR2055-481-2 \\
\hline IR50 & 1979 & Irrigated & IR9224-117-2-3-3-2 & IR2153-14-1-6-2/IR28//IR36 \\
\hline IR52 & 1980 & Irrigated & IR5853-118-5 & Nam Sa-gui 19/IR2071-88//IR2061-214-3-6-20 \\
\hline IR54 & 1980 & Irrigated & IR5853-162-1-2-3 & Nam Sa-gui 19/IR2071-88//IR2061-214-3-6-20 \\
\hline IR56 & 1982 & Irrigated & IR13429-109-2-2-1 & IR4432-53-33/PTB33//IR36 \\
\hline IR58 & 1983 & Irrigated & IR9752-71-3-2 & IR28/Kwang-chang-ai//IR36 \\
\hline IR60 & 1983 & Irrigated & IR13429-299-2-1-3 & IR4432-53-33/PTB33//IR36 \\
\hline IR62 & 1984 & Irrigated & IR13525-43-2-3-1-3-2 & PTB33/IR30//IR36 \\
\hline IR64 & 1985 & Irrigated & IR18348-36-3-3 & IR5657-33-2-1/IR2061-465-1-5-5 \\
\hline IR65 & 1985 & Irrigated & IR21015-196-3-1-3 & Batatais/IR36//IR52 \\
\hline IR66 & 1987 & Irrigated & IR32307-107-3-2-2 & IR13240-108-2-2-3/IR9129-209-2-2-2-1 \\
\hline IR68 & 1988 & Irrigated & IR28224-3-2-3-2 & IR19660-73-4/IR2415-90-4-3-2//IR54 \\
\hline IR70 & 1988 & Irrigated & IR28228-12-3-1-1-2 & IR19660-73-4/IR54//IR9828-36-3 \\
\hline IR72 & 1988 & Irrigated & IR35366-90-3-2-1-2 & IR19661-9-2-3/IR1576-199-3-3//IR9129-209-2-2-2-1 \\
\hline IR74 & 1988 & Irrigated & IR32453-20-3-2-2 & IR19661-131-1-2/IR15795-199-3-3 \\
\hline PSBRc1 & 1990 & Upland & IR10147-113-5-1-1-5 & KN-1B-361-1-8-6/IR1750-F5B-3//BPI76*9/Dawn \\
\hline PSBRc2 & 1991 & Irrigated & IR32809-26-3-3 & IR4215-301-2-2-6/BG90-2//IR19661-131-1-2 \\
\hline PSBRc4 & 1991 & Irrigated & IR41985-111-3-2-2 & IR4547-4-1-2/IR1905-81-3-1//IR25621-94-3-2 \\
\hline PSBRc10 & 1992 & Irrigated & IR50404-57-2-2-3 & IR33021-39-2-2/IR32429-47-3-2-2 \\
\hline PSBRc18 & 1994 & Irrigated & IR51672-62-2-1-1-2-3 & IR24594-204-1-3-2-6-2/IR28222-9-2-2-2-2 \\
\hline PSBRc20 & 1994 & Irrigated & IR57301-195-3-3 & IR35293-125-3-2-3/IR32429-47-3-2-2/PSBRc4 \\
\hline PSBRc28 & 1995 & Irrigated & IR56381-139-2-2 & IR28239-94-2-3-6-2/IR64 \\
\hline PSBRc30 & 1955 & Irrigated & IR58099-41-2-3 & IR72/IR24632-34-2 \\
\hline
\end{tabular}

Modified from data of IRRI ${ }^{11}$ and Peng $\& \mathrm{Khush}^{22}$. 


\section{Estimation of blast resistance genes}

The blast resistance gene(s) in the IRRI varieties were estimated following the reaction patterns of 9 kinds of monogenic lines ${ }^{26}$ to 14 blast isolates from the Philippines. Each monogenic line carries a different single resistance gene, Pi20, Pita, Pik-s, Pia, Pib, Piz-t, Pii, $P i 3$, and one of the Pik alleles (other than $P i k-s$ ). In the pathogenicity tests of blast isolates, $P i k$-s could be distinguished from the other four Pik alleles (Pik, Pik-h,Pik-m, and $P i k-p)$. However, no isolates were available to classify the four alleles ${ }^{25,29}$. The four alleles were designated tentatively as $P i k^{\dagger}$ in this study.

The IRRI-bred varieties were classified into several variety groups with distinct reaction patterns based mainly on the presence of three genes, Pi20, Pita and Pik $k^{\dagger}$ These three genes displayed more specific spectrums of resistance than those of other genes. These genes showed incompatibility with three blast isolates, M36-1-3-10-1 (avirulent to Pi20), IK81-25 (avirulent to Pita) and PO6-6 (avirulent to $P i k^{\dagger}$ ). Furthermore, classifying them into subgroups was carried out based on the presence of $P i b$, Pik-s, Piz-t, and Pii or Pi3.

\section{Gene identification based on segregation analysis using $\mathrm{BC}_{1} \mathrm{~F}_{2}$ family lines and allelism test}

$\mathrm{BC}_{1} \mathrm{~F}_{2}$ family lines and allelism tests were used to identify the blast resistance genes that have been estimated in the previous analysis by reaction patterns. Segregation analyses were performed using backcross progenies following a method proposed by Toriyama et $\mathrm{al}^{25}$, and allelism tests of the genes using $\mathrm{F}_{2}$ populations derived from crosses of resistant varieties with differential varieties. In both analyses, selected blast isolates with known avirulence and virulence were used to discriminate the resistance genes.

Six varieties, IR34, IR36, IR60, IR74, IR46, and IR64, were backcrossed with a susceptible Indica-type variety, CO39, as the recurrent parent and the developed $\mathrm{BC}_{1} \mathrm{~F}_{2}$ populations consisted of 53 to 140 lines. Each line, consisting of 20 seedlings, was inoculated with selected blast isolates avirulent to the particular genes. Following the procedure proposed by Toriyama et al. ${ }^{25}$, the number of genes conferring resistance in a variety was estimated from the segregation of $\mathrm{BC}_{1} \mathrm{~F}_{2}$ lines to each blast isolate. These segregation ratios of $1: 1,3: 1$ and $7: 1$ between segregating resistant and homozygously susceptible lines were expected for one, two and three genes controlling resistance, respectively. Each $\mathrm{BC}_{1} \mathrm{~F}_{2}$ line was analyzed for its reaction pattern to the blast isolates, and the kind of gene conferring the resistance was identified.

Nine varieties, IR34, IR24, IR36, IR60, PSBRc1, IR74, IR56, IR70, and IR64, were crossed with differen- tial varieties, which were known to be harboring resistance genes. $\mathrm{F}_{2}$ populations derived from each cross combination were used to confirm the genes. Selected blast isolates avirulent to these genes were inoculated to the seedlings of the same populations. As the differential varieties, Toride1 for Piz-t; BL1 for Pib; Yashiromochi, C105TTP2L9 and C101PKT for Pita; Tsuyuake with Pik$m$ and Kanto 51 with $P i k$ for $P i k^{\dagger}$; and IR24 for Pib, Pik-s, and Pi20 were used. The allelic relationship of the genes was determined based on the segregation of the $\mathrm{F}_{2}$ progenies of a cross to a particular blast isolate. If the segregation did not include susceptible plants, it was assumed that both parents had the same or an allelic gene. If otherwise, the resistance was governed by different genes.

\section{Results}

\section{Estimation of resistance genes}

The 42 varieties were classified into seven variety groups, VG1 to VG7. In some cases, subgroups within a group were also formed whenever the variety reacted differently to a particular isolate. Three variety groups, VG1, VG2 and VG7, were further divided into two, three and two subgroups, respectively. The number of varieties in each group varied from 1 to 17 (Table 2).

VG1: This group was characterized by the absence of the three genes Pi20, Pita and Pik $k^{\dagger}$. Seven varieties, IR20, IR28, IR29, IR30, IR34, IR45, and IR66, which showed a susceptible (S) reaction to M36-1-3-10-1 (avirulent to Pi20) and PO6-6 (avirulent to Pik') were included. All the varieties were resistant (R) to four isolates, BN209, V850196, B90002, and C923-49. These results suggested that two genes, Pib and Pik-s, were commonly present in VG1. Among these varieties, IR29 and IR34 additionally showed an $\mathrm{R}$ reaction to four isolates, IK81-25, IK81-3, M39-1-3-8-1, and M64-1-3-9-1. These reactions to M391-3-8-1 and M64-1-3-9-1 were attributed to the presence of Piz- $t$, while those to the other two isolates, IK81-25 and IK81-3, were to unknown gene(s). Based on these results, the VG1 group was reclassified into two subgroups, VG1a: IR20, IR28, IR30, IR45, and IR66, and VG1b: IR29 and IR34.

VG2: Seven varieties, IR8, IR22, IR24, IR26, PSBRc30, IR43, and PSBRc2, were included in this group harboring Pi20. They showed R reaction to isolate M361-3-10-1, which was avirulent to Pi20, and S reaction to two isolates, IK81-25 and PO6-6, which were virulent to Pi20. Additionally, all the varieties showed an $\mathrm{R}$ reaction to three isolates, BN209, B90002 and C923-49, which were avirulent to $P i b$. These results indicated that all the varieties in the VG2 group were likely to harbor Pib.

The VG2 group was divided into three subgroups, 


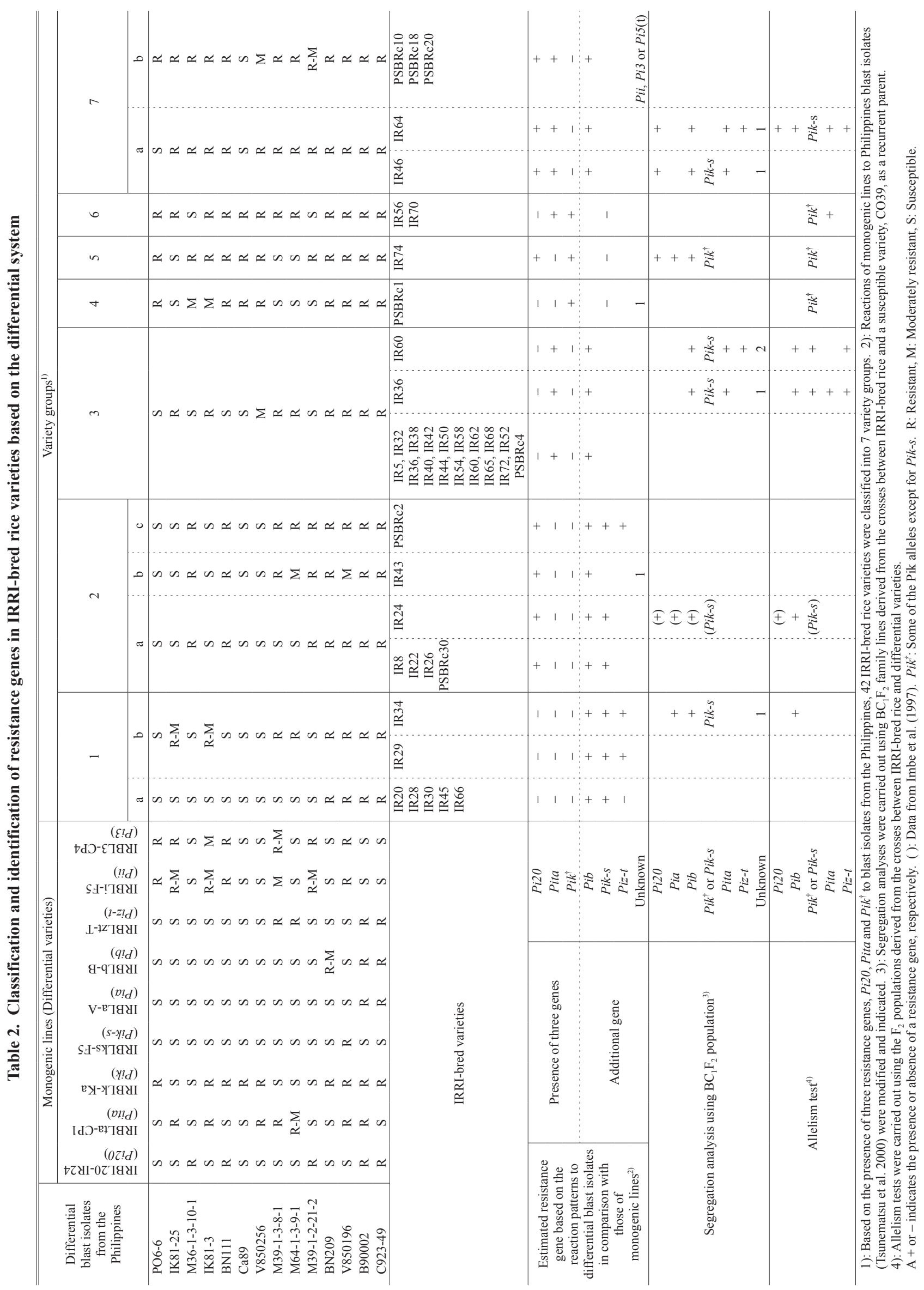


VG2a, VG2b and VG2c, differentiated by the reactions to three isolates, M39-1-3-8-1, M64-1-3-9-1 or V850196. The varieties in two subgroups, VG2a and VG2c, showed $\mathrm{R}$ reaction to $\mathrm{V} 850196$ whereas the $\mathrm{VG} 2 \mathrm{~b}$ variety displayed a moderate resistance to it. Pita and $P i k^{\dagger}$ were not present among VG2 varieties, and the $\mathrm{R}$ reaction to V850196 was estimated to be associated with the presence of Pik-s. The moderate resistance to the same isolate in VG2b could be attributed to the presence of an unknown gene. A variety PSBRc2 in $\mathrm{VG} 2 \mathrm{c}$ was resistant to both isolates, M39-1-3-8-1 and M64-1-3-9-1, and was estimated to harbor Piz-t. The VG2b variety showed a resistance to only M39-1-3-8-1 and a moderate resistance to M64-1-3-9-1. These kinds of resistance could not be associated with the reaction patterns in the differential system using the 14 blast isolates.

VG3: This group contained the largest number of varieties characterized by the presence of Pita. Seventeen varieties, IR5, IR32, IR36, IR38, IR40, IR42, IR44, IR50, IR52, IR54, IR58, IR60, IR62, IR65, IR68, IR72, and PSBRc4, were included. The absence of Pi2O and $P i k^{\dagger}$ in the varieties was estimated by their $\mathrm{S}$ reaction mainly to isolates M36-1-3-10-1 and PO6-6. In addition to the resistance to IK81-25, the presence of Pita in VG3 varieties was postulated by resistance to isolates that are also avirulent to Pita, namely, M39-1-3-8-1, M64-1-3-9-1 and IK81-3. All the varieties in this group also showed an R reaction to two isolates, BN209 and C923-49. Since these isolates were avirulent to $P i b$, it was estimated that this gene was also present in this group.

VG4: This group was characterized by $P i k^{\dagger}$ and only one variety, PSBRc1, was classified. PSBRc1 was moderately resistant to M36-1-3-10-1 but was susceptible to M39-1-2-21-2 and IK81-25 indicating that PSBRc1 does not harbor the other genes, Pi20 and Pita. Although isolate BN111 is avirulent to Pi20, this gene could not be estimated in the variety but only $P i k^{\dagger}$. It also showed moderate resistance to isolate IK81-3 that carries avirulence to $P i k^{\dagger}$ and Pita. It was estimated that additional unknown gene(s) conferred the moderate resistance in this variety.

VG5: Only IR74 was included in this group, which was estimated to harbor two genes, $P i k^{\dagger}$ and Pi20. The S reaction to IK81-25 indicated the absence of Pita in the variety. Two other isolates, M39-1-3-8-1 and M64-1-3-91, with known avirulence to Pita also exhibited the susceptibility of IR74.

VG6: This group was characterized by the presences of Pita and Pik $k^{\dagger}$ and included two varieties, IR56 and IR70. Although the resistance of IR56 and IR70 corresponded nearly to the reaction patterns of Pita and Pik the reaction of the varieties to isolate V850256 ranged from moderate resistance (M) to $\mathrm{R}$.

VG7: This group characterized by Pi20 and Pita, was divided into two subgroups, VG7a and VG7b. All 7 varieties also showed $\mathrm{R}$ reaction to isolate BN209, suggesting that the VG7 varieties carry Pib. The two subgroups were differentiated by the reaction to isolate PO66. The VG7a varieties were susceptible and the VG7b varieties were resistant to the isolate. The $\mathrm{R}$ reaction was estimated to be associated with one of the two genes, Pii or Pi3.

\section{Gene identification based on segregation analysis using $\mathrm{BC}_{1} \mathrm{~F}_{2}$ family lines}

The number and kinds of blast resistance genes in six varieties, IR34 in VG1b, IR36 and IR60 in VG3, IR74 in VG5, and IR46 and IR64 in VG7a, were analyzed using the $\mathrm{BC}_{1} \mathrm{~F}_{2}$ populations derived from the crosses between IRRI varieties and a susceptible Indica-type variety CO39. Four to six resistance genes, unknown included, were detected among these varieties (Table 3).

In IR34 three genes, Pib, Pik-s and Piz-t, were previously estimated. A total of $104 \mathrm{BC}_{1} \mathrm{~F}_{2}$ lines were examined for segregation to five isolates, M39-1-3-8-1, M64-13-9-1, BN209, V850196, and B90002. Against M391-3-8-1 and M64-1-3-9-1, the lines segregated into 48 segregating resistant and 56 homozygously susceptible lines, and a co-segregation for resistance was recognized between these isolates. The segregation fit a 1:1 ratio expected for a single dominant gene control. Since both isolates were avirulent to Piz-t and Pita, and IR34 was not previously estimated with Pita, the segregation for resistance was governed by Piz-t. To isolate BN209, the segregation of segregating resistant and homozygously susceptible lines fit a 3:1 ratio, suggesting that two dominant genes controlled the resistance. One of the genes was estimated as $P i b$ because this gene was incompatible with BN209, but the other one could not be identified based on the reaction pattern to blast isolates. Similarly, it was found that the resistance to an isolate V850196 was controlled by two dominant genes. One of these genes was estimated as Pik-s, but the other one could not be identified. The same lines were also examined for Pia, a gene that was not estimated directly by reaction pattern because it was masked by Pi20, Pita or Pik ${ }^{\dagger}$. All the lines were found to show $\mathrm{R}$ reaction to an isolate $\mathrm{B} 90002$, which was avirulent to Pia. Tsunematsu et al. ${ }^{26}$ indicated that the recurrent backcrossed parent, CO39, had Pia. Based on these results, IR34 was also confirmed with Pia. Thus, the presence of Pib, Pik-s and Piz-t was identified in IR34, while another gene Pia and at least another unknown one were also detected.

Two varieties, IR36 and IR60, previously estimated 


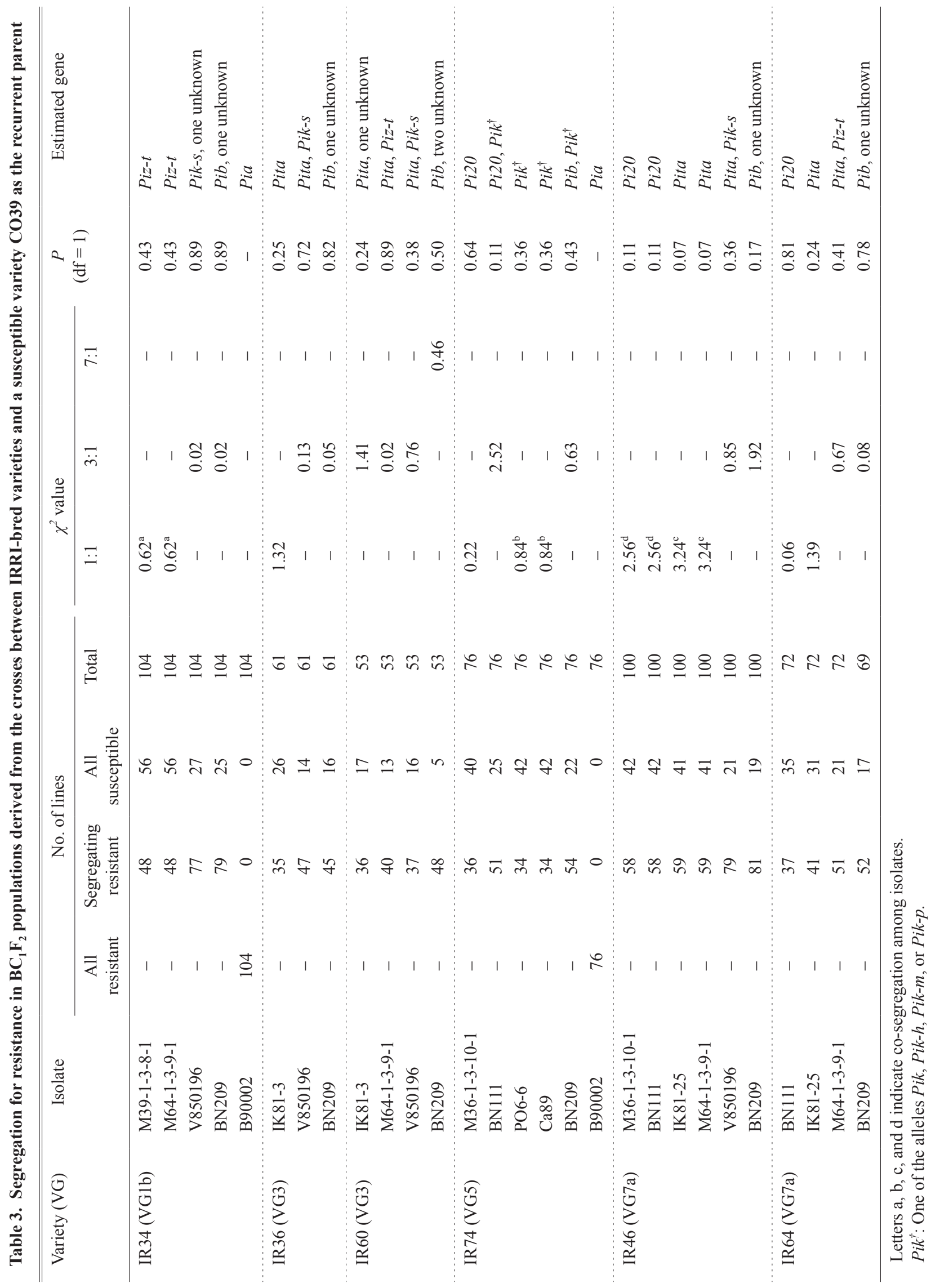


to harbor Pita and Pib were analyzed. Results showed that Pita could be identified in IR36 using isolates IK813, IK81-25 and V850196 which are avirulent to Pita. The $61 \mathrm{BC}_{1} \mathrm{~F}_{2}$ lines derived from a cross between IR36 and CO39 exhibited single gene segregation to IK81-3. The segregation for resistance was due to Pita. Two other genes, $P i k-s$ and Pib, were also identified in IR36 based on the segregation controlled by two dominant genes to V850196 which has avirulence to Pik-s and Pita; and to BN209 which has avirulence to $P i b$ and $P i k^{\dagger}$. Previous analysis showed that $P i k^{\dagger}$ was not included in VG3; hence, segregation for resistance to BN209 was related to Pib and another unknown gene. Therefore, the three genes Pib, Pita and Pik-s were confirmed in IR36, and an unknown one was also detected.

The presence of two genes, Pita and Pib, was also analyzed in IR0. Using $53 \mathrm{BC}_{1} \mathrm{~F}_{2}$ lines, a digenic segregation to each of the three isolates, IK81-3, M64-1-3-9-1 and V850196, was obtained as well as a trigenic one to BN209. IK81-3 was avirulent to Pita and Pik but the latter was not included in VG3. M64-1-3-9-1 has avirulence to Pita and Piz-t, V850196 to Pita and Pik-s, and BN209 to $P i b$; thus, the three genes Pita, Pik-s, and Pib were related in the segregation. However, one gene for resistance to IK81-3 and two genes for resistance to BN209 could not be identified by reaction patterns. With these findings, the presence of Pita and Pib was confirmed in IR60 besides Pik-s and Piz-t. IR60, therefore, harbors at least four genes.

In IR74 of VG5, two genes $P i 20$ and $P i k^{\dagger}$ were previously estimated and analyzed on $76 \mathrm{BC}_{1} \mathrm{~F}_{2}$ lines for segregation to 6 blast isolates: PO6-6 and Ca89 for identifying $P i k^{\dagger}$; M36-1-3-10-1 for Pi20; BN111 for Pi20 and Pik'; BN209 for $P i b$ and $P i k^{\dagger}$; and B90002 for Pia, Pik $k^{\dagger}, P i 20$, $P i b$, and Piz-t. The resistance was controlled by a single dominant gene based on the segregation ratios involving three isolates, PO6-6, Ca89 and M36-1-3-10-1, and a cosegregation was also observed between isolates PO6-6 and Ca89. Pik $k^{\dagger}$ controlled the segregation to PO6-6 and Ca89, while Pi20 to M36-1-3-10-1. A digenic segregation was observed for each to isolates BN111 and BN209. One of the genes, $P i k^{\dagger}$, was identified to be common in the segregation to both isolates. The other genes segregating to BN111 and BN209 corresponded to Pi20 and Pib, respectively. In the analysis of segregation to B90002, all the lines were resistant suggesting that IR74 carries the same Pia found in CO39. These results revealed that besides $P i 20$ and $P i k^{\dagger}$ identified so far in IR74, two other genes, $P i a$ and $P i b$ are present in this variety.

Two varieties, IR46 and IR64, were analyzed to confirm the presence of Pi20 and Pita. In the analysis of IR46 involving $100 \mathrm{BC}_{1} \mathrm{~F}_{2}$ lines and four isolates, $\mathrm{K} 81-25$,
M64-1-3-9-1, BN111, and M36-1-3-10-1, the segregation was controlled by a single dominant gene, and the co-segregation for resistance between BN111 and M36-1-3-101, and to IK81-25 and M64-1-3-9-1 were observed. Since BN111 and M36-1-3-10-1 are avirulent to Pi20, and IK8125 and M64-1-3-9-1 to Pita, the existence of Pita and Pi20 in IR46 was confirmed. The segregation for resistance to isolates V850196 and BN209 was controlled by two dominant genes. V850196 was avirulent to Pita, $P i k^{\dagger}$ and $P i k-s$, and BN209 to $P i b$ and $P i k^{\dagger}$. The gene $P i k^{\dagger}$ was not previously estimated in VG7a and that other genes, Pita and Pik-s, were related to the segregation for resistance to V850196, and $P i b$ and an unknown gene to BN209. Thus, Pi20, Pita, Pib, and Pik-s, and one unknown gene, are included in IR46.

In IR64, $72 \mathrm{BC}_{1} \mathrm{~F}_{2}$ lines were analyzed using four isolates, BN111, IK81-25, M64-1-3-9-1, and BN209. The segregation controlled by a single dominant gene was observed for isolates BN111 and IK81-25, and the segregation controlled by two dominant genes was observed for isolates M64-1-3-9-1 and BN209. Isolate BN111 is avirulent to Pi20 and Pik $k^{\dagger}$, IK81-25 to Pita, M64-1-3-9-1 to Pita and Piz-t, and BN209 to Pib and Pik ${ }^{\dagger}$. The gene $P i k^{\dagger}$ was not estimated among the varieties in VG7. From these results, four genes, Pita, Pib, Pi20, and Piz-t, were detected to be controlling the segregation for resistance to the isolates used. However, one of the two resistance genes could not be identified in the segregation analysis to BN209.

\section{Gene confirmation by allelism tests using differential varieties}

A total of $24 \mathrm{~F}_{2}$ populations derived from crosses among nine varieties and differential varieties were used for the allelism tests (Table 4).

In IR34, two $\mathrm{F}_{2}$ populations derived from the crosses with Toride1 for Piz-t, and BL1 for Pib, did not show any susceptible plants to isolates M64-1-3-9-1 and M39-1-3$8-1$, and BN209, respectively. The former two isolates and the latter one are avirulent to Piz- $t$ and Pib, respectively. From these results, it was confirmed that IR34 harbors Piz- $t$ and Pib.

In IR24, all $\mathrm{F}_{2}$ plants derived from the cross with BL1 for $P i b$ were resistant to BN209 that is avirulent to $P i k^{\dagger}$ and $P i b$. These results indicated the presence of $P i b$ in IR24. The existence of $P i b$ in IR24 previously reported by Imbe et al. ${ }^{10}$ was supported by the present allelism test.

In IR36, the four kinds of $\mathrm{F}_{2}$ populations derived from the crosses with C105TTP2L9 and C101PKT for Pita, Toride1 for Piz-t, and BL1 for Pib, segregated only resistant plants to isolates IK81-25, which is avirulent to 
Table 4. Allelism test for resistance using $F_{2}$ populations derived from the crosses between IRRI-bred and differential varieties

\begin{tabular}{|c|c|c|c|c|c|c|c|c|c|}
\hline \multirow{4}{*}{$\begin{array}{l}\text { Variety } \\
\text { (VG) } \\
\text { IR34 } \\
\text { (VG1b) }\end{array}$} & \multirow{2}{*}{\multicolumn{2}{|c|}{$\begin{array}{l}\text { Cross combination } \\
\text { (Resistance gene harbored in parent) }\end{array}$}} & \multirow{3}{*}{$\begin{array}{c}\text { Isolate } \\
\text { M39-1-3-8-1 }\end{array}$} & \multicolumn{3}{|c|}{ No. of plants ${ }^{a)}$} & \multirow{3}{*}{$\frac{\chi^{2} \text { value }}{(3: 1)}$} & \multirow{3}{*}{$\begin{array}{c}P \\
(\mathrm{df}=1) \\
-\end{array}$} & \multirow{3}{*}{$\begin{array}{l}\text { Identified gene } \\
\text { Piz-t }\end{array}$} \\
\hline & & & & \multirow{2}{*}{$\begin{array}{l}\mathrm{R} \\
94\end{array}$} & \multirow{2}{*}{$\frac{\mathrm{S}}{0}$} & \multirow{2}{*}{$\begin{array}{r}\text { Total } \\
94\end{array}$} & & & \\
\hline & Toride1 $($ Piz- $t)$ & / IR34 (Pia, Pik-s, Pib, Piz-t) & & & & & & & \\
\hline & & & M64-1-3-9-1 & 84 & 0 & 84 & - & - & $P i z-t$ \\
\hline & $\mathrm{BL} 1(P i b)$ & / IR34 & BN209 & 437 & 0 & 437 & - & - & $P i b$ \\
\hline $\begin{array}{l}\text { IR24 } \\
\text { (VG2b) }\end{array}$ & BL1 & / IR34 & BN209 & 161 & 0 & 161 & - & - & $P i b$ \\
\hline \multirow{9}{*}{$\begin{array}{l}\text { IR36 } \\
\text { (VG3) }\end{array}$} & IR36 (Pita, Pib, Pik-s) & / C105TTP2L9 (Pita) & $\mathrm{IK} 81-25$ & 468 & 0 & 468 & - & - & Pita \\
\hline & IR36 & / C101PKT (Pita) & IK81-25 & 167 & 0 & 167 & - & - & Pita \\
\hline & BL1 & / IR36 & BN209 & 420 & 0 & 420 & - & - & $P i b$ \\
\hline & IR24 & / IR36 & V850196 & 295 & 0 & 295 & - & - & Pik-s \\
\hline & & & BN209 & 287 & 0 & 287 & - & - & $P i b$ \\
\hline & Toride1 & / IR36 & M39-1-3-8-1 & 184 & 0 & 184 & - & - & $P i z-t$ \\
\hline & & & M64-1-3-9-1 & 168 & 0 & 168 & - & - & Piz- $t$ \\
\hline & IR34 & / IR36 & M39-1-3-8-1 & 467 & 0 & 467 & - & - & Piz-t \\
\hline & & & M64-1-3-9-1 & 516 & 0 & 516 & - & - & $P i z-t$ \\
\hline \multirow{4}{*}{$\begin{array}{l}\text { IR60 } \\
\text { (VG3) }\end{array}$} & IR34 & / IR60 (Pita, Pib, Pik-s, Piz-t) & M39-1-3-8-1 & 302 & 0 & 302 & - & - & Piz- $t$ \\
\hline & & & M64-1-3-9-1 & 303 & 0 & 303 & - & - & Piz- $t$ \\
\hline & IR24 & / IR60 & V850196 & 235 & 0 & 235 & - & - & Pik-s \\
\hline & & & BN209 & 360 & 0 & 360 & - & - & $P i b$ \\
\hline \multirow{3}{*}{$\begin{array}{l}\text { PSBRc1 } \\
\text { (VG4) }\end{array}$} & IR56 (Pita,Pik $\left.{ }^{\dagger}\right)$ & / PSBRc1 $\left(P i k^{\dagger}\right)$ & PO6-6 & 531 & 0 & 531 & - & - & $P i k^{\dagger}$ \\
\hline & & & BN111 & 526 & 0 & 526 & - & - & $P i k^{\dagger}$ \\
\hline & & & IK81-25 & 406 & 126 & 532 & 0.51 & 0.43 & Pita in IR56 \\
\hline \multirow{3}{*}{$\begin{array}{l}\text { IR74 } \\
\text { (VG5) }\end{array}$} & IR56 & / IR74 $\left(P i 20, P i k^{\dagger}\right)$ & PO6-6 & 560 & 0 & 560 & - & - & $P i k^{\dagger}$ \\
\hline & & & BN111 & 585 & 0 & 585 & - & - & $P i k^{\dagger}$ \\
\hline & & & IK81-25 & 442 & 135 & 577 & 0.82 & 0.37 & Pita in IR56 \\
\hline \multirow{8}{*}{$\begin{array}{l}\text { IR56 } \\
\text { (VG6) }\end{array}$} & C101PKT & / IR56 & IK81-25 & 469 & 0 & 469 & - & - & Pita \\
\hline & & & PO6-6 & 358 & 85 & 443 & 7.98 & 0.005 & $P i k^{\dagger}$ in IR56 \\
\hline & Yashiromochi (Pita) & / IR56 & IK81-25 & 463 & 0 & 463 & - & - & Pita \\
\hline & & & PO6-6 & 370 & 127 & 497 & 0.08 & 0.78 & $P i k^{\dagger}$ in IR56 \\
\hline & IR56 & / Kanto51 (Pik) & PO6-6 & 440 & 0 & 440 & - & - & $P i k^{\dagger}$ \\
\hline & & & BN111 & 407 & 0 & 407 & - & - & $P i k^{\dagger}$ \\
\hline & IR56 & / Tsuyuake $(P i k-m)$ & PO6-6 & 498 & 0 & 498 & - & - & $P i k^{\dagger}$ \\
\hline & & & BN111 & 496 & 0 & 496 & - & - & $P i k^{\dagger}$ \\
\hline \multirow{3}{*}{$\begin{array}{l}\text { IR70 } \\
\text { (VG6) }\end{array}$} & IR56 & / IR70 (Pita, Pik $\left.{ }^{\dagger}\right)$ & IK $81-25$ & 567 & 0 & 567 & - & - & Pita \\
\hline & & & PO6-6 & 577 & 0 & 577 & - & - & $P i k^{\dagger}$ \\
\hline & & & BN111 & 558 & 0 & 558 & - & - & $P i k^{\dagger}$ \\
\hline \multirow{9}{*}{$\begin{array}{l}\text { IR64 } \\
(\text { VG7a) }\end{array}$} & IR24 & / IR64 (Pi20, Pita, Pib) & $\mathrm{BN} 111$ & 302 & 0 & 302 & - & - & $P i 20$ \\
\hline & & & V850196 & 227 & 0 & 227 & - & - & Pik-s \\
\hline & & & BN209 & 222 & 0 & 222 & - & - & $P i b$ \\
\hline & IR64 & / C105TTP2L9 & IK81-25 & 274 & 0 & 274 & - & - & Pita \\
\hline & IR64 & / C101PKT & IK81-25 & 132 & 0 & 132 & - & - & Pita \\
\hline & IR56 & / IR64 & IK81-25 & 296 & 0 & 296 & - & - & Pita \\
\hline & & & PO6-6 & 193 & 60 & 253 & 0.22 & 0.64 & $P i k^{\dagger}$ in IR56 \\
\hline & Toride1 & / IR64 & M39-1-3-8-1 & 124 & 0 & 124 & - & - & $P i z-t$ \\
\hline & & & M64-1-3-9-1 & 133 & 0 & 133 & - & - & Piz-t \\
\hline
\end{tabular}

a): $\mathrm{R}=$ resistant, $\mathrm{S}=$ susceptible. 
Pita, M39-1-3-8-1 and M64-1-3-9-1 to Pita and Piz-t, and BN209 to $P i b$, respectively. These results confirmed that IR36 has Pita, Piz- $t$ and Pib. The presence of Piz- $t$ was also confirmed from the segregation of the $\mathrm{F}_{2}$ population derived from a cross between IR34 and IR36 using isolates M39-1-3-8-1 and M64-1-3-9-1. In the $\mathrm{F}_{2}$ population derived from a cross between IR24 and IR36, the allelism of $P i b$ and Pik-s was confirmed using isolates BN209 and V850196, respectively.

The $\mathrm{F}_{2}$ populations derived from the crosses of IR60 with IR34 and IR24 did not segregate any susceptible plants to isolates BN209 avirulent to Pib and $P i k^{\dagger}$, V850196 to Pita, Pik-s and Pik ${ }^{\dagger}$, and M39-1-3-8-1 and M64-1-3-9-1 to Pita and Piz-t. IR34 was confirmed to have Pia, Pib, Piz-t, and Pik-s, and IR24 has Pia, Pib, $P i k-s$, and Pi20. IR60 was not previously estimated to have $P i k^{\dagger}$ while the resistance to M39-1-3-8-1 and M641-3-9-1 was related to Piz-t which was estimated also in IR34. On this basis, the three genes Pib, Piz-t and Pik-s were also confirmed in IR60 of VG3.

In PSBRc1, the $\mathrm{F}_{2}$ populations derived from a cross with IR56 previously estimated to have Pik ${ }^{\dagger}$ and Pita, showed that all plants were resistant to PO6-6 avirulent to $P i k^{\dagger}$, and BN111 avirulent to Pik $k^{\dagger}$ and Pi20. Since Pi20 was not estimated in PSBRc1 by the reaction pattern in previous analysis, the resistance of the $\mathrm{F}_{2}$ plants segregated into 406 resistant and 126 susceptible which fits a $3: 1$ ratio $\left(\chi^{2}=0.51, P=0.43\right)$. This would indicate that a dominant gene Pita estimated in IR56 governed this segregation.

In IR74, all $\mathrm{F}_{2}$ plants derived from a cross with IR56 showed resistance to PO6-6 avirulent to $P i k^{\dagger}$, and BN111 to $P i k^{\dagger}$ and $P i 20$ which confirmed that IR74 has the same $P i k^{\dagger}$ allele of IR56. On the other hand, segregation to IK81-25 avirulent to Pita fit to a 3:1 ratio $\left(\chi^{2}=0.82, P=\right.$ $0.37)$ expected for a single dominant gene control of resistance due to Pita in IR56.

IR56 was crossed with five differential varieties, C101PKT and Yashiromochi for Pita, Kanto51 and Tsuyuake for Pik $k^{\dagger}$, and IR70 for Pita and Pik ${ }^{\dagger}$. Eleven kinds of segregation analyses were carried out using three isolates: IK81-25 avirulent to Pita, PO6-6 to Pik ${ }^{\dagger}$, and BN111 to $P i k^{\dagger}$ and Pi20. Of these, two $\mathrm{F}_{2}$ populations derived from crosses with C101PKT and Yashiromochi showed a single gene segregation to PO6-6. The other nine populations did not segregate any susceptible plants suggesting that the same gene controlled the resistance. It was estimated that the segregation to PO6-6 was due to $P i k^{\dagger}$ in IR56. Since the Philippine blast isolates could not classify the $P i k$ alleles except $P i k-s$, no susceptible plants were obtained from the populations derived from the combinations among Kanto51, Tsuyuake and IR56 for $P i k^{\dagger}$.
These results confirmed that IR56 harbors Pita and Pik ${ }^{\dagger}$.

The $\mathrm{F}_{2}$ populations derived from the crosses between IR64 and differential varieties, C105TTP2L9 and C101PKT for Pita, Toride1 for Piz- $t$ and IR24 for Pi20 did not show any susceptible plants to IK81-25, M39-1-38-1, M64-1-3-9-1, and BN111. These results indicated that IR64 had the same alleles of Pita, Piz-t and Pi20. A similar reaction was shown to IK81-25 in the $F_{2}$ plants from a cross between IR64 and IR56. However, there was a single gene segregation $\left(\chi^{2}=0.22, P=0.64\right)$ to PO6-6 due to $P i k^{\dagger}$ in IR56. Against V850196 and BN209, the $\mathrm{F}_{2}$ population of a cross of IR64 with IR24 did not segregate any susceptible plants indicating that the same genes $P i k-S$ and $P i b$ conferred resistance to both isolates. These results confirmed that $P i k-s$ and $P i b$ were also included in IR64.

These six resistance genes, Pi20, Pik ${ }^{\dagger}$, Pita, Pib, Pik$s$, and Piz-t, were confirmed by the allelism test.

\section{Discussion}

\section{Origins of resistance genes in IRRI-bred varieties}

At least 8 kinds of blast resistance genes, Pia, Pib, Pi20, Pita, Pik ${ }^{\dagger}$, Pik-s, Piz-t, and Pii or Pi3(t), were estimated in 42 IRRI-bred rice varieties through the estimation by reaction patterns to blast isolates from the Philippines, gene identification using $\mathrm{BC}_{1} \mathrm{~F}_{2}$ population and gene confirmation by allelism tests, based on the differential system. Among the seven blast resistance genes, $P i b$ and $P i k$ alleles, $P i k-s$ and $P i k^{\dagger}$, were identified in almost all varieties (Table 5). These wide distributions of the two genes might be correlated with the breeding method applied for developing these varieties. Most often, these varieties included IR8, IR24 and IR36, or their hybrid progenies in their pedigrees.

IR8 was bred from a cross between an Indonesian variety, Peta, and Dee-geo-woo-gen from Taiwan. Peta and Dee-geo-woo-gen were estimated to carry Pib and $P i k-s$, respectively (unpublished data). IR8 and its progenies were found in the pedigrees of IR24 and IR36. Three varieties, Dawn, Tadukan and Tetep could also be found in the pedigrees of IRRI-bred varieties. They were estimated to harbor a Pik allele (other than Pik-s) (unpublished data). Kiyosawa ${ }^{16}$ reported that $P i k$ - $h$ was identified in these varieties. On the basis of these findings, Dee-geo-woo-gen and the three varieties, Dawn, Tadukan and Tetep, could be the sources of Pik-s and Pikं, respectively. It will be necessary to confirm the genotypes of $P i k^{\dagger}$ using blast isolates which can classify the four alleles, Pik, Pik-h, Pik-m, and Pik-p. These findings suggested that Peta or Dee-geo-woo-gen might be the sources of Pib gene of the IRRI varieties. 


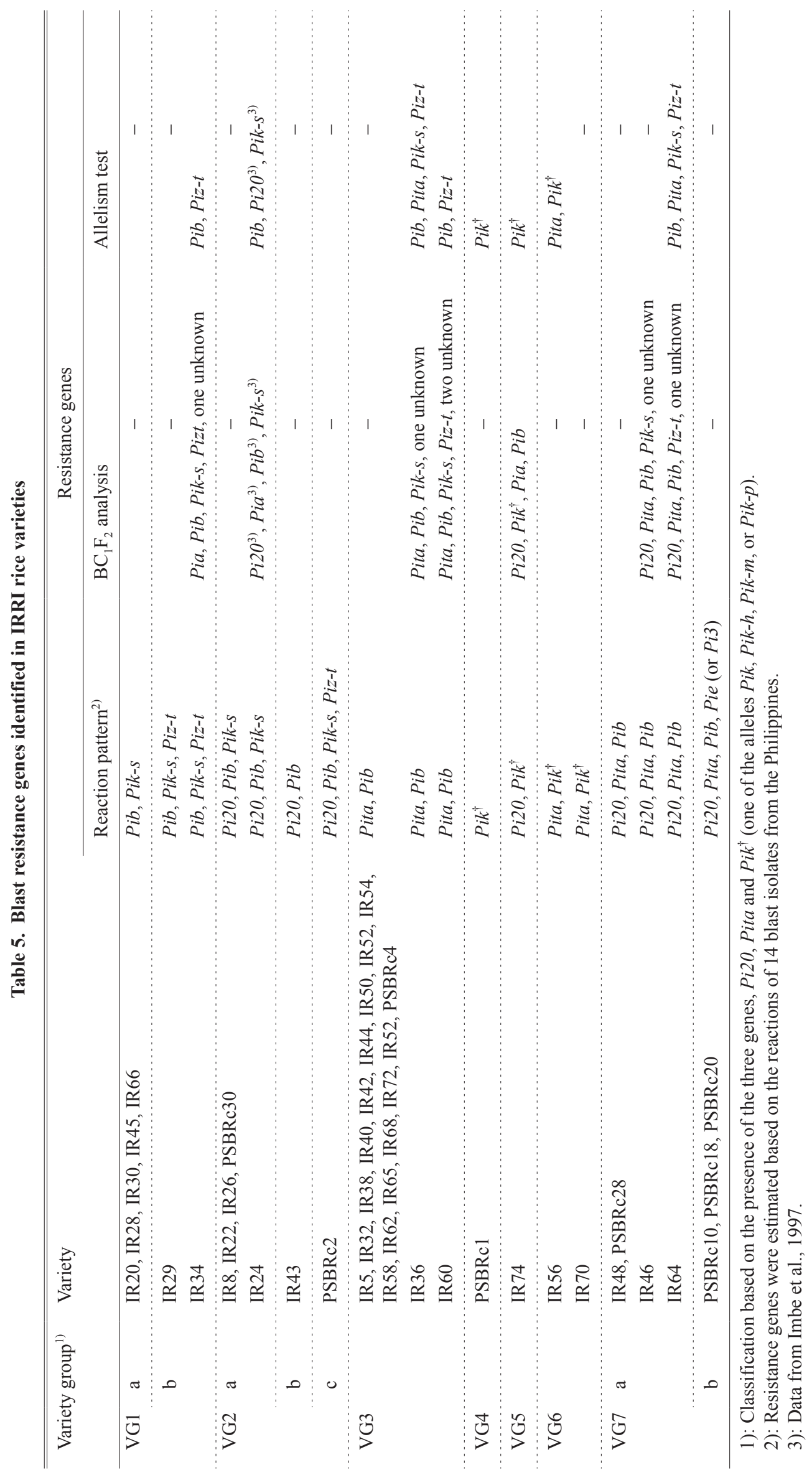


Among 42 varieties, 15 harbored Pi20 and these were classified into three groups, VG2, VG5 and VG7. IR8 and its derived progeny, IR24, were included in VG2. All the other varieties in VG2 were bred from the crosses with IR8, IR24 or Peta. In the other groups, these three varieties and their progenies were also found in the pedigrees of the varieties. Imbe et al. ${ }^{10}$ found Pi20 in IR24. Other than Peta, variety Sigadis, was also included in the pedigree of IR24. Peta and Sigadis were estimated to harbor Pi20 (unpublished data). These results suggested that Pi20 originated from Peta or Sigadis in the early stage of the IRRI breeding activities.

Pita was harbored in the varieties of the largest group of VG3. IR36, once the most popular variety ${ }^{11}$, and its sister lines were found in the pedigrees of these varieties. IR36 was selected from the progeny of IRRI breeding line IR2071 derived from crosses of the progenies of IR24 and IR8. The five varieties, IR32, IR38, IR40, IR42, and IR44, were also selected from IR2071 and IR2070 crosses that had the same parental line, CR94-13. As for the other varieties, IR50, IR52, IR54, IR58, IR60, IR62, IR65, IR68, and IR72 have IR36, IR2071 cross, or IR36 hybrid progenies that were included in their pedigrees. In the case of more recent varieties from other groups harboring Pita, such as IR56 and IR70 in VG6, and IR64, PSBRc10, PSBRc18, PSBRc20, and PSBRc28 in VG7, these also have IR36 or its progenies in their pedigrees. IR36 was probably responsible for transmitting Pita into these varieties. A line, CR94-13, was believed to carry Pita, which is effective against Philippine blast isolates (unpublished data). Kiyosawa ${ }^{14}$ reported that a rice variety from the Philippines, Tadukan, harbored Pita. Tadukan was also used in the breeding of IR36. Based on these findings, CR94-13 or Tadukan could be the source of Pita in IRRI varieties.

Yokoo and Kiyosawa ${ }^{32}$ reported that Piz- $t$ conferred a broad resistance spectrum against the races of blast fungus and was transmitted by an Indian-type variety, TKM1. With the use of blast isolates from the Philippines, Piz- $t$ was estimated in TKM6, which was one of the lines in the TKM breeding series (unpublished data). The presence of Piz- $t$ was estimated in IR29 and IR34 belonging to VG1b and PSBRc2 belonging to VG2c. TKM6 and its crossed line IR1561 were included in the pedigrees of these three varieties. Although IR28 in the VG1a subgroup is a sister variety of IR29 and IR34, it failed to inherit Piz-t. Thus, TKM6 was estimated to be a source of Piz- $t$ of these varieties.

Pia was also identified in IR34 and IR74. Imbe et al. ${ }^{10}$ had already reported the presence of Pia in IR24. Many IRRI varieties had IR24 or its hybrid progenies as a parental variety in their pedigrees. The source of Pia was estimated to have originated from Blue Bonnet ${ }^{15}$, and such a finding could very well support the existence of this gene and its wide distribution to many IRRI varieties.

Estimations of the presence of Pib, Pik-s, Piz-t, and Pia were not easy whenever Pi20, Pita and Pik $k^{\dagger}$ were carried in the same varieties. These three genes, Pi20, Pita and $P i k^{\dagger}$, show a relatively wide spectrum of resistance and they can mask the reactions of the narrow spectrum genes, Pia, Pib, Pik-s, and Piz-t. In this study, segregation analysis using backcross progenies and allelism tests were demonstrated to be useful methods for identifying these genes. It will be necessary to use these methods for confirmation with the remaining varieties which were not attempted in these segregation analyses because the analyzed varieties had limited numbers among IRRI-bred ones.

\section{Characterization of IRRI-bred varieties based on the blast resistance genes}

The presence of limited numbers of blast resistance genes were clarified in the IRRI-bred varieties. Hargrove et al. ${ }^{6}$ suggested that it might be due to the genetic relatedness among these varieties. For example, IR8, IR24 and IR36, or their progenies were often used during the development of IRRI rice breeding. The presences of blast resistance genes can be also characterized in these IRRIbred varieties. The resistance genes among leading varieties, IR8, IR24, IR36, IR64, and IR72, indicated the unique process of IRRI breeding. IR8 and IR24 have the common three resistance genes, Pib, Pi20 and Pik-s, and were included in the same group, VG2a. IR24 is a progeny of the cross between IR8 and IR127, and IR24 is expected to inherit a lot of traits from IR8. In IR36, the Pi20 was removed and two new genes, Pita and Piz-t were added. However, IR8 and IR24 were included in IR36's pedigree; the findings suggested that new resources were used that caused a dramatic change to occur in the breeding. In IR64, these five genes, Pib, Pi20, Pik-s, Pita, and $P i z$ - $t$ were pyramided. These results suggest that the germplasm improvement had been carried out with the bases of IR 8 and IR24 genetic background in IRRI breeding. IR72 was found to have only two genes, Pib and Pita, and the next dramatic change was expected to be tried in the breeding.

This study is the first study for elucidating the genetic constitution for blast resistance of important Indica-type IRRI-bred rice varieties based on the differential system governed by a gene-for-gene relationship between rice and the blast pathogen. This information on these blast resistance genes will be useful towards breeding for durable resistance to blast whenever IRRI varieties are utilized. 


\section{Acknowledgments}

We would like to thank former Professor Masao Yokoo and Associate Professor Ryo Ohsawa of Tsukuba University for their support, as well as Drs. Gurdev S. Khush, David J. Mackill, Tokio Imbe, Shinjo Koizumi, Hiroshi Kato, Hiroshi Tsunematsu, and Etsuko Araki, for their valuable support and encouragement.

\section{References}

1. Ebron, L. A. et al. (2004) Estimation of genes in blast resistance in elite Indica-type rice (Oryza sativa L.) varieties-bred at International Rice Research Institute. Breed. Sci., 54, 381-387.

2. Ebron, L. A. et al. (2005) Identification of blast resistance genes in elite Indica-type varieties of rice (Oryza sativa L.). SABRAO J. Breed. Genet., 37(1), 19-31.

3. Flor, H. H. (1971) Current status of the gene-for-gene concept. Annu. Rev. Phytopathol., 9, 275-296.

4. Flores-Gaxiola, J. et al. (1983) Inheritance of blast Pyricularia oryzae resistance in rice. Int. Rice Res. Newsl., 8, 5-6.

5. Fukuta, Y. et al. (2004) Development of differential varieties for blast resistance in IRRI-Japan collaborative research project. In Rice blast: Interaction with rice and control, Proc. 3rd International rice blast conference, ed. Kawasaki, S., Kluwer Academic Publishers, Netherlands, 229-233.

6. Hargrove, T. R., Coffman, W. R. \& Cabanilla, V. L. (1979) Genetic interrelationships of improved rice varieties in Asia. IRRI research paper series No. 23. IRRI, Manila, Philippines.

7. Hossain, M. (1995) Sustaining food security for fragile environments in Asia: achievements, challenges and implications for rice research. In Proceedings of the international rice research conference, IRRI, Manila, Philippines, 3-23.

8. Imbe, T. \& Matsumoto, S. (1985) Inheritance of resistance of rice varieties to the blast fungus strains virulent to the variety 'Reiho'. Jpn. J. Breed., 35, 332-339 [In Japanese with English summary].

9. Imbe, T. (1997) Stabilization of rice culture under water stress in the tropics utilizing a broader spectrum of genetic resources, progress report (December 1995-November 1996). IRRI-Government of Japan Collaborative Research Project.

10. Imbe, T. et al. (1997) A new gene for blast resistance in rice cultivar IR24. Rice Genet. Newsl., 14, 60-62.

11. International Rice Research Institute (1982) IR36, the world's most popular rice. IRRI, Manila, Philippines.

12. Khush, G. S. (1987) Rice breeding: past, present and future. J. Genet., 66, 195-216.

13. Khush, G. S. (1990) Varietal needs for different environments and breeding strategies. In New frontiers in rice research, eds. Muralidharan, K. \& Siddiq, E. A., Directorate of Rice Research, Hyderabad, India, 68-75.

14. Kiyosawa, S. (1966) Studies on inheritance of resistance of rice varieties to blast. 3. Inheritance of a rice variety $\mathrm{Pi}$
No. 1 to the blast fungus. Jpn. J. Breed., 16, 243-250.

15. Kiyosawa, S. (1972) Genetics of blast resistance. In Rice breeding, IRRI, Manila, Philippines, 203-225.

16. Kiyosawa, S. (1981) Gene analysis for blast resistance. Oryza, 18, 196-203.

17. Kropff, M. J. et al. (1994) Quantitative understanding of yield potential. In Breaking the yield barrier, proceedings of a workshop on rice yield potential in favorable environments, ed. Cassman, K. G., International Rice Research Institute, Manila, Philippines, 21-38.

18. Mackill, D. J. \& Bonman J. M. (1992) Inheritance of blast resistance in near-isogenic lines of rice. Phytopathology, 82, 746-749.

19. Noda, T. et al. (1999) Distribution of pathogenic races of rice blast fungus in Vietnam. Ann. Phytopathol. Soc. Jpn., 65, 526-530.

20. Notteghem, J. L. et al. (1994) Present knowledge of rice resistance genetics and strategies for Magnaporthe grisea pathogenicity and virulence gene analysis. In Rice blast disease, eds. Zeigler, R. S., Leong, S. A. \& Teng, P. S., CAB International, Wallinford, 155-166.

21. Pedersen, W. L. \& Leath, S. (1988) Pyramiding major genes for resistance to maintain residual effects. Annu. Rev. Phytopathol., 26, 369-378.

22. Peng, S. \& Khush, G. S. (2003) Four decades of breeding for varietal improvement of irrigated lowland rice in the International Rice Research Institute. Plant Prod. Sci., 6(3), 157-164.

23. Roumen, E. C. (1992) Small differential interactions for partial resistance in rice cultivars to virulent isolates of the blast pathogen. Euphytica, 64, 143-148.

24. Sallaud, C. et al. (2003) Identification of five new blast resistance genes in the blast-resistant rice variety IR64 using a QTL mapping strategy. Theor. Appl. Genet., 106, 794-803.

25. Toriyama, K. et al. (1986) A method of estimating true resistance genes to blast in rice varieties. In Rice genetics, International Rice Research Institute, Manila, Philippines, 441-449.

26. Tsunematsu, H. et al. (2000) Development of monogenic lines of rice for blast resistance. Breed. Sci., 50, 229-234.

27. Wang, Z., Mackill, D. J. \& Bonman, J. M. (1989) Inheritance of partial resistance to blast in Indica rice cultivars. Crop Sci., 29, 848-853.

28. Yamada, M. et al. (1976) Proposal of a new method for differentiating races of Pyricularia oryza Cavara in Japan. Ann. Phytopathol. Soc. Jpn., 42, 216-219.

29. Yamaguchi, M. et al. (1996) Genetic analysis for true resistance to blast in rice varieties. Int. Rice Res. Notes, 21(2-3), 51-52.

30. Yanoria, M. J. T. et al. (2000) Pathogenicity of IRRI blast isolates to rice blast resistance genes. Poster presented at the 4th International Rice Genetics Symposium, October 22-27, 2000, International Rice Research Institute, Manila, Philippines.

31. Yeh, W. H. \& Bonman, J. M. (1986) Assessment of partial resistance to Pyricularia oryzae in six rice cultivars. Plant Pathol., 35, 319-323.

32. Yokoo, M. \& Kiyosawa, S. (1970) Inheritance of blast resistance of the rice variety, Toride 1 , selected from the 
cross Norin 8 x TKM 1. Jpn. J. Breed., 3, 129-132.

33. Yu, Z. H., Mackill, D. H. \& Bonman, J. M. (1987)
Inheritance of resistance to blast in some traditional and improved rice cultivars. Phytopatholy, 77, 323-326. 